\title{
Cancer-associated thromboembolism: could direct- acting oral anticoagulants be a promising therapeutic option? Literature review
}

\begin{abstract}
Background: Cancer-associated thromboembolism (CAT) is usually managed with low-molecular-weight heparins (LMWHs) or vitamin $\mathrm{K}$ antagonists (VKAs). The recent data suggest that direct-acting oral anticoagulants (DOACs) might have a role in the management of CAT both in terms of prevention and treatment. The aim of this article is to review the current literature regarding the use of DOACs in patients with cancer.

Methods: PUBMED and ClinicalTrials.gov were searched to identify prospective trials on patients with cancer managed with DOACs as either primary prophylaxis or treatment of venous thromboembolic events.

Results: In terms of primary prophylaxis, five studies (CASSINI, ADVOCATE, MYELAXAT, NCT02958969, and AVERT ) have been identified. When considered together, CASSINI and AVERT showed a significant benefit of rivaroxaban and apixaban, respectively, versus placebo for the prevention of venous thromboembolism (VTE), with a low incidence of major bleeding and no significant difference in mortality. ADVOCATE, MYELAXAT, and NCT02958969 showed that apixaban was well tolerated in patients with metastases receiving chemotherapy or those with multiple myeloma receiving immunomodulatory imide drugs. With respect to VTE treatment, 3 studies (HOKUSAI-VTE, SELECT-D, and ADAM-VTE ) were identified. These studies demonstrated significantly lower rates of VTE recurrences in patients treated with edoxaban, rivaroxaban, or apixaban, without compromise on safety.

Conclusions: DOACs seem a promising therapeutic option in CAT. As cancer is a heterogeneous disease, future research will clarify the role of these newer anticoagulant agents in both prevention and treatment of cancer-related VTE.
\end{abstract}

Keywords: cancer • venous thromboembolic event $\bullet$ CAT $\bullet$ direct oral anticoagulant drugs $\bullet$ DOACs

\section{Introduction}

Cancer is one of the most common and important acquired risk factor for venous thromboembolism (VTE) $[1,2]$ and patients with active malignancy have a fourfold to sevenfold higher incidence of symptomatic VTE than the general population $[1,3,4]$.

Since thromboembolism has been found to be a leading cause of death in patients with cancer, recent guidelines [5-8] have addressed the issue of VTE treatment, as well as VTE primary prophylaxis, recommending mainly the standard anticoagulant regimens used in these patients (heparins and vitamin $\mathrm{K}$ antagonists [VKAs]). At present, low-molecularweight heparins (LMWHs) represent the cornerstone of anticoagulant treatment and are endorsed by many international guidelines [5-8]. However, the requirement of daily subcutaneous injections, the problematic use in renal insufficiency and the rare but often fatal heparininduced thrombocytopenia (HIT) syndrome are some of the obstacles that limit their use.

The introduction of direct oral anticoagulants (DOACs) has raised strong expectations, because they potentially represent an attractive alternative to 
VKAs and LMWHs. DOACs do not require laboratory monitoring or daily injections and appear to be costsaving options. In the large, noninferiority, randomized controlled trials DOACs were compared with LMWH/ VKAs and demonstrated similar efficacy but superior safety in the treatment of acute symptomatic VTE [9]. These findings were consistent in clinically important subgroups. "Active cancer," representing $6 \%$ of the total population, was one of the subgroups in which DOACs demonstrated efficacy and safety similar to those of the standard treatment. However, the definition of active cancer differed among the trials, and limited data were available in regard to the type of cancer, the staging of disease, and the oncologic treatment of patients. Most importantly, DOACS were compared with VKAs and not to long-term LMWHs, which represent the preferable treatment in patients with cancer. Therefore, an unmet need in this therapeutic area was to conduct studies that would test the efficacy and safety of DOACs versus LMWHs in a population with cancer.

The aim of this article is to provide an overview of the prospective trials, which were specifically conducted in patients with cancer treated with DOACs versus the standard or no treatment, either for primary prophylaxis or VTE treatment.

\section{Guidelines}

Routine pharmacological thromboprophylaxis is recommended in patients with cancer admitted to hospital for medical or surgical reasons during hospitalization and up to 4 weeks in high-risk operations. In ambulatory patients with cancer, routine thromboprophylaxis is not recommended [5-7] but may be considered for highly selected high-risk patients (mainly those with metastatic pancreatic cancer receiving chemotherapy) [8]. In this context, outpatient multiple myeloma patients receiving thalidomide or lenalidomide regimens with chemotherapy and/or dexamethasone should receive thromboprophylaxis with LMWH, warfarin (higher-risk patients), or aspirin (lower-risk patients) [5,6]. Use of DOACs for prevention of VTE in patients with cancer is not yet recommended [6].

VTE treatment should be initially (5-10 days) treated with LMWH [6] or unfractioned heparin (UFH) $[5,7]$. LMWH is the first-choice regimen for long-term treatment (3-6 months) [5,6], with VKA as an alternative choice if LMWH is not available [6,7]. However, the latest NCCN guidelines [7] have included DOACs among the available treatment choices for VTE. Monotherapy options include rivaroxaban (Category $2 \mathrm{~A}$ ) and apixaban (Category $2 \mathrm{~A}$ ); the latter is for those patients who refuse or have compelling reasons to avoid LMWH. Combination therapy options include LMWH + edoxaban (Category 1) or UFH + edoxaban (Category 2A) and $\mathrm{LMWH}+$ dabigatran (Category 2A) or UFH + dabigatran (Category 2A). It is, nonetheless, stated that urinary or gastrointestinal (GI) tract lesions, pathology, or instrumentation are relative contraindications to the use of DOACs in cancer. In addition, the European Labelling Information for DOACs states that the efficacy and safety of apixaban, dabigatran, and edoxaban have not been established in the treatment of deep vein thrombosis (DVT) or pulmonary embolism (PE), as well as in the prevention of recurrent DVT/PE in patients with active cancer, whereas no information is provided for rivaroxaban.

VTE treatment should be given for at least 6 months and individualized thereafter, because there is no consensus about the maximum treatment period $[6,7]$.

\section{DOACs in primary thromboprophylaxis}

\subsection{Rivaroxaban}

In the CASSINI trial [10], 841 high-risk (Khorana score of $\geq 2$ ) ambulatory patients with cancer were randomly assigned to receive either rivaroxaban $10 \mathrm{mg}$ once daily or placebo for up to 180 days, with screening every 8 weeks. The primary efficacy end point of this double-blind trial was a composite of objectively confirmed proximal DVT in a lower limb, PE, symptomatic DVT in an upper limb or distal DVT in a lower limb, and VTE-related death. The primary safety end point was major bleeding. The primary efficacy end point occurred in $6.0 \%$ of the patients in the rivaroxaban group and in $8.8 \%$ of those in the placebo group $(\mathrm{HR}=0.66 ; \mathrm{P}=0.10)$, with more than one-third of events to occur after the discontinuation of study drug. Therefore, a prespecified interventionperiod analysis was performed and demonstrated that the primary end point occurred in $2.6 \%$ of the patients in the rivaroxaban versus $6.4 \%$ of those in the placebo group ( $\mathrm{HR}=0.40 ; 95 \% \mathrm{Cl}: 0.20-0.80)$, pointing out to a difference of 4 percentage points in favor of rivaroxaban with regard to the composite of VTE and VTE-related death. This was translated to an absolute risk reduction (ARR) of $3.8 \%$ corresponding to a number needed to treat (NNT) of 26 during the interventionperiod analysis. Major bleeding occurred in $2.0 \%$ of the patients in the rivaroxaban group and in $1.0 \%$ of those in the placebo group ( $\mathrm{HR}=1.96 ; 95 \% \mathrm{Cl}$ : 0.59-6.49). Clinically relevant non-major (CRNM) bleeding occurred in $2.7 \%$ of the patients in the rivaroxaban group and in 
$2.0 \%$ of those in the placebo group $(\mathrm{HR}=1.34 ; 95 \%$ $\mathrm{Cl}$ : 0.54-3.32). In conclusion, in high-risk ambulatory patients with cancer, treatment with rivaroxaban did not result in a significantly lower incidence of venous thromboembolism or death due to VTE. However, during the intervention period, rivaroxaban led to a substantially lower incidence of such events, with a low incidence of major bleeding. Furthermore, the lower rate of both arterial and distal asymptomatic events with rivaroxaban than with placebo, although not included in the primary end point, could further increase the net benefit of prophylaxis for these patients.

\subsection{Apixaban}

The ADVOCATE trial [11] randomized 130 ambulatory patients undergoing first-line or second-line chemotherapy for advanced or metastatic cancer to receive prophylactically apixaban $(5,10$, and $20 \mathrm{mg}$ once daily) or placebo for 12 weeks, beginning within 4 weeks of the start of chemotherapy. The primary outcome of this phase II trial was the composite of major or CRNM bleeding, whereas the secondary outcomes were symptomatic DVT/PE, grade 3 or higher adverse events (AEs), or death. The primary outcome occurred in $6.5 \%$ of patients in the apixaban group versus $3.4 \%$ of those in the placebo group. The rate of major bleeding in patients given apixaban was low at $2.2 \%(95 \% \mathrm{Cl}$ : $0.26 \%-0.75 \%$ ) versus $3.4 \%$ in the placebo group, and most of these events occurred in the 20-mg group. Three patients $(10.3 \%)$ in the placebo group and none in the apixaban groups developed symptomatic DVT or PE. In this phase II trial, 12 weeks of apixaban, when compared with placebo, appeared to be well tolerated for the prevention of VTE in ambulatory patients undergoing first-line or second-line chemotherapy for advanced or metastatic cancer.

In the MYELAXAT pilot study [12], 104 patients with multiple myeloma treated with immunomodulatory imide drugs (IMiD) received apixaban in a preventive scheme (2.5 mg twice daily) for 6 months. All patients were in first-line treatment (11) or in relapse (93) and required the prevention of VTE events with aspirin or LMWH. The primary end points were VTE and VTE-related death, as well as major and CRNM bleeding within 7 months. The secondary end points included the incidence of venous thromboembolic complications according to the thrombotic risk stratification and the time of treatment with iMiDs (diagnosis or relapse), as well as the incidence of bleedings and arterial cardiovascular events (MI, ischemic stroke, and TIA). No PE or arterial cardiovascular events were reported, whereas two venous thrombotic events were registered (incidence: $0.38 \%$ patient-month), although apixaban was discontinued 14 days before, because of lenalidomideinduced thrombopenia. Only one major and 11 CRNM hemorrhages were reported. Referring to the incidence of thromboembolic events in Carrier's meta-analysis [13] and to hemorrhagic events in medical patients receiving apixaban in primary VTE prophylaxis [14], apixaban used in a preventive scheme seems to be efficient and safe in preventing VTE in patients with myeloma treated with IMiD compounds.

More positive data on multiple myeloma prophylaxis have been available recently [15]. Data from the 3-month interim analysis of a pilot, phase IV, singlearm study involving 50 patients with multiple myeloma receiving immunomodulatory and apixaban $2.5 \mathrm{mg}$ twice daily as thromboprophylaxis showed that no patients experienced VTE, major hemorrhage, stroke, or myocardial infarction. Two patients experienced CRNM hemorrhage. In this pilot study of 50 patients, low-dose apixaban was safe and well tolerated as thromboprophylaxis for patients with MM receiving IMiDs, but further randomized studies are needed to validate apixaban as a standard primary prevention antithrombotic strategy for these patients.

In the apixaban for the prevention of venous thromboembolism in high-risk ambulatory patients with cancer (AVERT) trial [16], the objective was to assess the efficacy of apixaban thromboprophylaxis in ambulatory patients with cancer at intermediate to high risk for VTE (Khorana score $\geq 2$ ), who were initiating chemotherapy. About 574 patients were randomized to receive apixaban $2.5 \mathrm{mg}$ twice daily or placebo for 6 months. The primary efficacy outcome was objectively documented VTE, and the main safety outcome was a major bleeding episode. VTE occurred in $4.2 \%$ of the patients in the apixaban group and in $10.2 \%$ of those in the placebo group $(\mathrm{HR}=0.41 ; \mathrm{P}<0.001)$, translating to a significant $59 \%$ relative risk reduction of major VTE with apixaban. The ARR was $6 \%$, corresponding to an NNT of 17 . With regard to safety, major bleeding occurred in significantly more patients receiving apixaban versus placebo in the modified intention-to-treat analysis $(3.5 \%$ vs $1.8 \%$, respectively) $(\mathrm{HR}=2.00 ; \mathrm{P}=0.046)$. However, when analysis was performed during the treatment period, major bleeding occurred in $2.1 \%$ of the patients who had actually received apixaban versus $1.1 \%$ of those in the placebo group ( $\mathrm{HR}=1.89 ; 95 \% \mathrm{Cl}: 0.39$ 9.24). No cases of fatal bleeding or bleeding into critical organs occurred in either group. Authors' conclusion was that apixaban resulted in a significantly lower rate of VTE than did placebo among moderate to high risk ambulatory patients with cancer, but significantly 
increased the rate of major bleedings. However, major bleedings were not significantly higher in the per-protocol analysis, which was the primary safety outcome.

\section{DOACs in VTE treatment}

\subsection{Edoxaban}

The HOKUSAI VTE trial [17] randomized 1,046 patients with cancer and acute symptomatic or incidental VTE to receive either edoxaban (at least 5 days of LMWHs followed by $60 \mathrm{mg}$ daily), or dalteparin (200 IU/kg daily for 1 month followed by $150 \mathrm{lU} / \mathrm{kg}$ daily) for at least 6 months and up to 12 months. The primary outcome of this open-label, noninferiority trial was a composite of recurrent VTE or major bleeding in the first 12 months. The primary outcome occurred in $12.8 \%$ of the patients in the edoxaban group and in $13.5 \%$ of those in the dalteparin group $(\mathrm{HR}=0.97 ; \mathrm{P}=0.006$ for noninferiority; $P=0.87$ for superiority), confirming the noninferiority of edoxaban. The rate of recurrent VTE was $7.9 \%$ in the edoxaban arm and $11.3 \%$ in the dalteparin arm (HR = $0.71 ; 95 \% \mathrm{Cl}: 0.48-1.06)$, again without a statistically significant difference. With regard to safety, the rate of major bleeding was significantly higher, $6.9 \%$ versus $4.0 \%$ for the edoxaban and dalteparin arms, respectively $(\mathrm{HR}=1.77 ; 95 \% \mathrm{Cl}: 1.03-3.04)$. This difference was mainly due to a higher rate of upper $\mathrm{GI}$ bleeding with edoxaban, occurring mainly in patients who had entered the trial with GI cancer. However, the frequency of severe major bleedings (considered as a clinical emergency) was similar between the two groups $(2.3 \%)$. No differences in terms of overall survival were observed between the groups. Author's conclusion was that oral edoxaban was noninferior to subcutaneous dalteparin with respect to the composite outcome of recurrent VTE or major bleeding. Edoxaban's lower rate of recurrent VTE was offset by an increased risk of major bleeding.

\subsection{Rivaroxaban}

In the SELECT-D pilot trial [18], 406 patients with active cancer who presented with symptomatic/incidental PE or symptomatic lower-extremity proximal DVT were randomly assigned to receive either rivaroxaban (15 mg twice daily for 21 days followed by $20 \mathrm{mg}$ daily) or dalteparin $(200 \mathrm{IU} / \mathrm{kg}$ daily for the first 30 days, followed by $150 \mathrm{IU} / \mathrm{kg}$ ) for a total of 6 months. The main objective of this study was to assess VTE recurrence and safety (rates of major bleedings and CRNMB) in the first 6 months of treatment. The second objective of the study, which aimed to assess the treatment duration beyond 6 months, remained unanswered because of low recruitment at the second planned randomization (lower assignment because of high mortality rate and clinician's decision). The 6-month cumulative VTE recurrence rate was $11 \%$ (95\% Cl: $7-16 \%)$ with dalteparin and 4\% (95\% $\mathrm{Cl}: 2-9 \%)$ with rivaroxaban $(\mathrm{HR}=0.43 ; 95 \% \mathrm{Cl}: 0.19-$ 0.99). The 6-month cumulative rate of major bleeding was $4 \%(95 \% \mathrm{Cl}: 2-8 \%)$ for dalteparin and $6 \%(95 \%$ Cl: 3-11\%) for rivaroxaban ( $\mathrm{HR}=1.83$; 95\% Cl: 0.684.96). Corresponding rates of CRNMB were $4 \%(95 \%$ Cl: $2-9 \%)$ and $13 \%$ (95\% Cl: 9-19\%), respectively (HR $=3.76$; 95\% Cl: 1.63-8.69), demonstrating a threefold relative increase in CRNMB with rivaroxaban compared with dalteparin. There were no central nervous system (CNS) bleeds and most of major bleeding was of $\mathrm{GI}$ origin occurring mainly in patients with esophageal or gastroesophageal cancer. No statistically significant differences in overall survival were observed in both the arms at 6 months (70\% for dalteparin and $75 \%$ for rivaroxaban). Author's conclusion was that rivaroxaban was associated with relatively low VTE recurrence but higher CRNMB when compared with dalteparin.

\subsection{Apixaban}

In the ADAM-VTE trial [19], which was a randomized, open-label study, patients with cancer-associated acute VTE were randomly assigned to receive either apixaban $10 \mathrm{mg}$ twice daily for 7 days followed by 5 mg twice daily or subcutaneous dalteparin (200 IU/ $\mathrm{kg}$ for 1 month followed by $150 \mathrm{lU} / \mathrm{kg}$ once daily) for 6 months. The primary outcome was major bleeding. The secondary outcomes included VTE recurrence and a composite of major plus CRNM bleeding. Of the 300 patients who underwent randomization, 287 were included in the primary analysis. Of these, metastatic disease was present in $65.5 \%$ of the patients and $74 \%$ were receiving concurrent systemic cancer therapy. Colorectal, lung, pancreas, and breast cancers were the four most prevalent cancer types. An interesting point was that ADAM-VTE did not exclude patients with brain metastases. Major bleeding occurred in $0 \%$ of the patients in the apixaban group when compared with $1.4 \%$ of those in the dalteparin group $(P=0.138)$. Recurrent VTE occurred in $0.7 \%$ of the patients in the apixaban group and in $6.3 \%$ of those in the dalteparin group ( $\mathrm{HR}=0.099 ; 95 \% \mathrm{Cl}: 0.013-0.780 ; \mathrm{P}=.0281)$. Major plus CRNM bleeding were similar at $6 \%$ for both the groups. No differences in mortality observed when comparing the apixaban (16\%) and dalteparin (11\%) 
groups at 6 months $(\mathrm{HR}=1.40 ; 95 \% \mathrm{Cl}: 0.82-2.43 ; \mathrm{P}=$ $0.3078)$ ), although this study was not powered to detect survival differences. Monthly quality-of-life surveys favored apixaban therapy for many measures including: concern for excess bruising, stress, irritation, burden of delivery, and overall satisfaction with anticoagulant therapy $(\mathrm{P}<0.05)$. Monthly bruising questionnaire favored apixaban at each interval $(P<0.002)$. During the trial, there were patients in both arms who refused further treatment. More patients randomized to dalteparin stopped therapy relative to the apixaban arm $(P=0.0012)$. Author's conclusion was that oral apixaban therapy was associated with very low rates of bleeding and significantly lower VTE recurrence with superior quality of life outcome measures, compared to dalteparin in the treatment of cancer associated VTE.

\section{Discussion}

Patients with cancer are at an increased risk of thromboembolic events. Current management of CAT consists mainly of LMWH. However, parenteral therapy adds stress and frustration to patients with cancer and negatively impacts their quality of life. Recent trials suggest that DOACs could possibly emerge as a new therapeutic option in the future.

In the cumulative analysis of CASSINI and AVERT by Agnelli et al [20], rivaroxaban and apixaban showed a significant benefit for the prevention of VTE, with a low incidence of major bleeding in ambulatory patients with cancer with a Khorana score of $\geq 2$. In addition, there was not a significant difference in the mortality between patients who received a DOAC and those who received placebo.

In the metanalysis of HOKUSAI-Cancer, SELECT-D, and ADAM-VTE trials, Brunetti et al [21] investigated the efficacy and safety of DOACs versus LMWH in VTE treatment. Studies were comparable for principal features, except for the follow-up duration. In this metanalysis, it was shown for the first time a significant superiority of DOACs versus LMWH in terms of lower recurrence of VTE in patients with cancer; this superiority in efficacy was not significantly counterbalanced by safety concerns in the presence of comparable bleeding events and mortality rates.
The results of both prevention and treatment metanalysis seem to favor DOACs at least in terms of efficacy and urge us to partly reconsider what was previously considered as the standard therapy in the oncologic population. There is no doubt that the key to prevention and treatment of cancer-associated VTE is adequate anticoagulation with a careful and individualized approach. This is more than true in the case of DOACs considering the different pharmacokinetics aspects (clearance through P-glycoprotein and/or cytochrome P450), the increased risk of bleeding in some cancer types (e.g., GI or urologic malignancy) and the considerable interaction with some antineoplastic drugs. Reduced renal function and extreme body weights are limiting factors for the use of both LMWHs and DOACs. The preferences and the values of the patient should also be included in the decision-making process of defining the type of anticoagulation.

We need trials that should take into account relevant features such as the cancer type and activity, the underlying antineoplastic treatment, the age, and comorbidities of patients. This would allow a more reliable assessment of the clinical benefit of DOACs. The results of such studies are eagerly awaited in the next few years. CASTA-DIVA; NCT02746185, CONKO-011; NCT02583191, Caravaggio; NCT03045406, PRIORITY; NCT03139487, CANVAS; NCT02744092, CAP; NCT02581176, NCT03240120, NCT02366871 are some of the studies that will hopefully shed light on the challenging topic of both VTE prophylaxis and VTE treatment in patients with cancer.

\section{Conclusion}

DOACs have the potential to improve the management of CAT by making it more efficient, equally safe, simpler, and less cumbersome compared with the standard treatment. However, further research in this complex setting is certainly warranted.

Conflicts of Interest: Paraskevi Savvari is currently working as Pfizer employee. 
1] KhoranaAAet al. Incidence and predictors of venous thromboembolism (VTE) among ambulatory highrisk cancer patients undergoing chemotherapy in the United States. Cancer. 2013; 119:648-655.

[2] Kessler CM. The link between cancer and venous thromboembolism: a review. Am J Clin Oncol. 2009;32: S3-S7

[3] Heit JA, Silverstein MD, Mohr DN, Petterson TM, O'Fallon WM, Melton LJ 3rd. Risk factors for deep vein thrombosis and pulmonary embolism: a population-based case-control study. Arch Intern Med. 2000; 160:809-815.

[4] Blom JW, Doggen CJ, Osanto S, Rosendaal FR. Malignancies, prothrombotic mutations, and the risk of venous thrombosis. JAMA. 2005; 293:715722.

[5] Mandalà M, Falanga A, Roila F; ESMO Guidelines Working Group. Management of venous thromboembolism (VTE) in cancer patients: ESMO Clinical Practice Guidelines. Ann Oncol. 2011;22:vi85-vi92

[6] ASCO 2015 Guidelines/VTE. Available at http:// www.asco.org/sites/new-www.asco.org/files/ content-files/practice-and-guidelines/documents/ VTE-Summary-of-Recs.pdf

[7] With permission from the NCCN Clinical Practice Guidelines in Oncology (NCCN Guidelines ${ }^{\circledR}$ ) for Cancer-Associated Venous Thromboembolic Disease V.2.2018 - August 27, 2018. (C) National Comprehensive Cancer all rights reserved. Accessed 10.12.2018. To view the most recent and complete version of the guideline, go online to WWW.NCCN.org

[8] National Institute for Health and Care Excellence (2018) Venous thromboembolism in over 16s: reducing the risk of hospital-acquired deep vein thrombosis or pulmonary embolism. NICE guideline (NG89). Available at https://www.nice.org.uk/ guidance/ng89. Last accessed December 2018

[9] van Es N, CoppensM, Schulman S, Middeldorp S, Büller HR. Direct oral anticoagulants compared with vitamin $\mathrm{K}$ antagonists for acute venous thromboembolism: evidence from phase 3 trials. Blood 2014;124(12):1968-1975

[10] Khorana AA. et al Rivaroxaban for Thromboprophylaxis in High-Risk Ambulatory Patients with Cancer. N Engl J Med 2019; 380:720728

[11] Levine MN, Gu C, Liebman HA, Escalante CP, Solymoss S, Deitchman D et al. A randomized phase II trial of apixaban for the prevention of thromboembolism in patients with metastatic cancer. J Thromb Haemost. 2012; 10:807-14.

[12] Pegourie B, Karlin L, Benboubker L, OrsiniPiocelle F, Tiab M, Auger-Quittet S et al. Apixaban for the prevention of thromboembolism in Immunomodulatory-treated myeloma patients: Myelaxat, a phase 2 pilot study. Am J Hematol. 2019;94(6):635-640

[13] Carrier M, Le Gal G, Tay J, Wu C, Lee AY. Rates of venous thromboembolism in multiple myeloma patients undergoing immunomodulatory therapy with thalidomide or lenalidomide: a systematic review and meta-analysis. J Thromb Haemost. 2011; 9:653-63.

[14] Goldhaber SZ, Leizorovicz A, Kakkar AK, Haas SK, Merli G, Knabb RM et al; ADOPT Trial Investigators. Apixaban versus enoxaparin for thromboprophylaxis in medically ill patients. N Engl J Med. 2011;365:2167-77.

[15] Cornell RF, Goldhaber SZ, Engelhardt BG, Moslehi J, Jagasia M, Patton D et al. Prospective Study of Apixaban for Primary Prevention of Venous Thromboembolism in Patients With Multiple Myeloma Receiving Immunomodulatory Therapy, a 3-Month Interim Analysis. ASH 2018. https://ash.confex.com/ash/2018/webprogram/ Paper112577.html (accessed January 14, 2019).

[16] Carrier M, Abou-Nassar K, Mallick R, Tagalakis V, Shivakumar S, Schattner A et al. Apixaban to Prevent Venous Thromboembolism in Patients with Cancer. N Engl J Med. 2019 21; 380:711-719

[17] Raskob GE, van Es N, Verhamme P, Carrier M, Di Nisio M, Garcia D et al. Edoxaban for the Treatment of Cancer-Associated Venous Thromboembolism. N Engl J Med.2018 15; 378:615-624.

[18] Young AM, Marshall A, Thirlwall J, Chapman O, Lokare A, Hill C et al. Comparison of an Oral Factor Xa Inhibitor with Low Molecular Weight Heparin in Patients With Cancer With Venous Thromboembolism: Results of a Randomized Trial (SELECT-D). J Clin Oncol. 2018 10; 36:2017-2023.

[19] McBane II R, Loprinzi CL, Le-Rademacher JG, Zemla T, Ashrani A, Tafur A. et al. Apixaban and Dalteparin in Active Malignancy Associated Venous Thromboembolism. The ADAM VTE Trial. J Thromb Haemost. 2019. [Epub ahead of print]

[20] Agnelli G. Direct Oral Anticoagulants for Thromboprophylaxis in Ambulatory Patients with Cancer. N Engl J Med. 2019 ;380(8):781-783 
[21] Brunetti ND, Tricarico L, Correale M, De Gennaro $\mathrm{L}$, Santoro F, leva R et al. Direct oral anticoagulants more effective than low-molecular-weight heparin for venous thrombo-embolism in cancer: an updated meta-analysis of randomized trials. J Thromb Thrombolysis. 2019. [Epub ahead of print] 